\title{
Analyzing Displacement Term's Memory Effect in a van der Pol Type Boundary Condition to Prove Chaotic Vibration of the Wave Equation
}

\author{
Goong Chen* \\ Department of Mathematics, Texas A\&M University \\ College Station, TX 77843, USA \\ Sze-Bi Hsu ${ }^{\dagger}$ \\ Department of Mathematics, National Tsing Hua University \\ Hsinchu 30043, Taiwan, R.O.C. \\ Tingwen Huang $\ddagger$ \\ Department of Mathematics, Texas A\&M University \\ College Station, TX 77843, USA
}

\begin{abstract}
Consider the one-dimensional wave equation on a unit interval, where the left-end boundary condition is linear, pumping energy into the system, while the right-end boundary condition is self-regulating of the van der Pol type with a cubic nonlinearity. Then for a certain parameter range it is now known that chaotic vibration occurs. However, if the right-end van der Pol boundary condition contains an extra linear displacement feedback term, then it induces a memory effect and considerable technical difficulty arises as to how to define and determine chaotic vibration of the system. In this paper, we take advantage of the extra margin property of the reflection map and utilize properties of homoclinic orbits coupled with a perturbation approach to show that for a small parameter range, chaotic vibrations occur in the sense of unbounded growth of snapshots of the gradient. The work also has significant implications to the occurrence of chaotic vibration for the wave equation on a $3 \mathrm{D}$ annular domain.
\end{abstract}

\section{Introduction}

First, we take this opportunity to express our great admiration toward Professor Leon O. Chua. Throughout his career so far, Professor Chua has made major contributions to many areas of electrical engineering, particularly, that of nonlinear circuits and systems. He has an unusual, high appreciation of the role played by mathematics in the research and development of applied sciences and technology. The journal founded by him, the International Journal of
Bifurcation and Chaos in Applied Sciences and Engineering (IJBC), has fully bloomed into a premier journal in nonlinear science under his editorship. It is also the most favorite forum for us to publish our mathematical papers on chaos. We just wish to say our appreciation, in a small way, "Thank you, Professor Chua", by dedicating this paper to him on the occasion of his $65^{\text {th }}$ birthday.

The problem we wish to address is the chaotic vibration of the wave equation. (Our first research

\footnotetext{
*Supported in part by DARPA grant F49620-01-1-0566. E-mail: gchen@math.tamu.edu

${ }^{\dagger}$ Supported in part by a grant from National Science Council of R.O.C. E-mail: sbhsu@math.nthu.edu.tw

${ }^{\ddagger}$ E-mail: twhuang@math.tamu.edu
} 
article on this subject, [Chen et al., 1996], was published by IJBC in 1996.) Consider the following. The wave equation

$$
\frac{\partial^{2} w(x, t)}{\partial x^{2}}-\frac{\partial^{2} w(x, t)}{\partial t^{2}}=0, \quad 0<x<1, \quad t>0,
$$

on a bounded interval $(0,1)$, where the speed of wave propagation is assumed to be one without loss of generality as far as the mathematical analysis herein is concerned. The boundary condition at the left-end satisfies

$$
w_{t}(0, t)=-\eta w_{x}(0, t), \quad \eta>0, \quad \eta \neq 1, \quad t>0,
$$

while that at the right-end satisfies

$$
\left.\begin{array}{l}
w_{x}(1, t)=\alpha w_{t}(1, t)-\beta w_{t}^{3}(1, t)-\gamma w(1, t), \\
t>0,0<\alpha<1, \beta>0, \gamma>0 .
\end{array}\right\}
$$

The initial conditions are given by

$$
w(x, 0)=w_{0}(x), \quad w_{t}(x, 0)=w_{1}(x), \quad 0<x<1 .
$$

Note that the boundary condition (1.2) signifies the pumping of energy into the system in a feedback way. The boundary condition (1.3) is similar to the van der Pol nonlinearity we studied in our earlier work [Chen et al., 1998a-1998d]. The major distinction here is the presence of the term $\gamma w$ in the RHS (right hand side) of (1.3). In elastic vibrations, this term $\gamma w$ usually corresponds to some elastic support of a vibrating string at $x=1$; see [Chen \& Zhou, 1993], for example. The rate of change of energy of the vibrating system is

$$
\begin{aligned}
& \frac{d}{d t} E(t) \\
\equiv & \frac{d}{d t}\left\{\frac{1}{2} \int_{0}^{1}\left[w_{x}^{2}(x, t)+w_{t}^{2}(x, t)\right] d x+\frac{\gamma}{2} w^{2}(1, t)\right\}
\end{aligned}
$$$$
=(\cdots \text { integration by parts and simplification, }
$$$$
\text { using (1.1)-(1.3)) }
$$$$
=\eta w_{x}^{2}(0, t)+\left[\alpha w_{t}^{2}(1, t)-\beta w_{t}^{4}(1, t)\right] .
$$

Since

$$
\begin{aligned}
& \eta w_{x}^{2}(0, t) \geq 0, \quad \text { for all } t>0, \\
& \alpha w_{t}^{2}(1, t)-\beta w_{t}^{4}(1, t) \\
& \begin{cases}\geq 0, & \text { if }\left|w_{t}(1, t)\right| \leq \sqrt{\alpha / \beta}, \\
<0, & \text { if }\left|w_{t}(1, t)\right|>\sqrt{\alpha / \beta},\end{cases}
\end{aligned}
$$

we see that (1.6) injects energy into the system while (1.7) is self-regulating just like the usual van der Pol nonlinearity treated in [Chen et al., 1998a-1998d].

Remark 1.1. How important is it to include the $\gamma w$ term in (1.3)? There are two major reasons that motivate us to consider it in this paper:

(1) In the standard PID (proportional, integral and differential) methodology of feedback control, the feedback of position or displacement is of utmost importance in problems such as tracking. Here the $\gamma w$ term corresponds precisely to the position or displacement term.

(2) The $\gamma w$ term may arise due to reduction of dimensionality for certain symmetry. Consider the following: The wave equation in a $3 \mathrm{D}$ annular domain $\Omega$ :

$$
\begin{aligned}
& \Delta W(\boldsymbol{x}, t)-\frac{1}{c^{2}} W_{t t}(\boldsymbol{x}, t)=0, \quad x \in \Omega, \quad t>0, \\
& \Omega=\{\boldsymbol{x}|a<| \boldsymbol{x} \mid<b\},
\end{aligned}
$$

where $a>0, b>0, x=\left(x_{1}, x_{2}, x_{3}\right)$, and $\Delta=$ $\frac{\partial^{2}}{\partial x_{1}^{2}}+\frac{\partial^{2}}{\partial x_{2}^{2}}+\frac{\partial^{2}}{\partial x_{3}^{2}}$. Let $n$ denote the outward unit normal vector on $\partial \Omega$, the boundary of $\Omega$. The boundary condition on the inner shell $|\boldsymbol{x}|=a$ is assumed to be

$$
\begin{gathered}
\frac{\partial W(\boldsymbol{x}, t)}{\partial n}=\alpha W_{t}(\boldsymbol{x}, t)-\beta W_{t}^{3}(\boldsymbol{x}, t)-k_{1} W(\boldsymbol{x}, t) \\
k_{1} \geq 0, \quad|x|=a, \quad t>0
\end{gathered}
$$

and that on the outer shell $|\boldsymbol{x}|=b$ is

$$
\begin{aligned}
\frac{\partial W(\boldsymbol{x}, t)}{\partial n}= & \frac{1}{\eta} W_{t}(\boldsymbol{x}, t)-k_{2} W(\boldsymbol{x}, t), \\
& \eta>0, \quad k_{2} \geq 0, \quad|x|=b, \quad t>0 .
\end{aligned}
$$


The initial conditions satisfy

$W(\boldsymbol{x}, 0)=W_{0}(|\boldsymbol{x}|), \quad W_{t}(\boldsymbol{x}, 0)=W_{1}(|\boldsymbol{x}|), \quad \boldsymbol{x} \in \Omega$,

for some functions $W_{0}$ and $W_{1}$ defined on $\mathbb{R}$. Let us again examine the rate of change of energy:

$$
\begin{aligned}
\frac{d}{d t} E(t) \equiv & \frac{d}{d t}\left\{\frac{1}{2} \int_{\Omega}\left[|\nabla W(\boldsymbol{x}, t)|^{2}+\frac{1}{c^{2}} W_{t}^{2}(\boldsymbol{x}, t)\right] d \boldsymbol{x}\right. \\
& +\frac{k_{1}}{2} \int_{|\boldsymbol{x}|=a} W^{2}(\boldsymbol{x}, t) d \sigma \\
& \left.+\frac{k_{2}}{2} \int_{|\boldsymbol{x}|=b} W^{2}(\boldsymbol{x}, t) d \sigma\right\} \\
= & \int_{\Omega}\left[\nabla W(\boldsymbol{x}, t) \cdot \nabla W_{t}(\boldsymbol{x}, t)\right. \\
& \left.+\frac{1}{c^{2}} W_{t}(\boldsymbol{x}, t) W_{t t}(\boldsymbol{x}, t)\right] d \boldsymbol{x} \\
& +k_{1} \int_{|\boldsymbol{x}|=a} W(\boldsymbol{x}, t) W_{t}(\boldsymbol{x}, t) d \sigma \\
& +k_{2} \int_{|\boldsymbol{x}|=b} W(\boldsymbol{x}, t) W_{t}(\boldsymbol{x}, t) d \sigma
\end{aligned}
$$

$=\cdots$ (integration by parts and simplification, utilizing (1.8)-(1.10))

$$
\begin{aligned}
& =\int_{|\boldsymbol{x}|=a}\left[\alpha-\beta W_{t}^{2}(\boldsymbol{x}, t)\right] W_{t}^{2}(\boldsymbol{x}, t) d \sigma \\
& +\frac{1}{\eta} \int_{|\boldsymbol{x}|=b} W_{t}^{2}(\boldsymbol{x}, t) d \sigma .
\end{aligned}
$$

In $(1.15)_{3}$ above, set

$$
k_{2}=\frac{1}{b}
$$

then we can eliminate the term $\eta\left(k_{2}-\frac{1}{b}\right) w(b, t)$. We further make the change of variable

$$
r=b-(b-a) x, \quad 0 \leq x \leq 1 .
$$

Then (1.15) becomes

$$
\left\{\begin{array}{l}
\frac{1}{(b-a)^{2}} w_{x x}(x, t)-\frac{1}{c^{2}} w_{t t}(x, t)=0 \\
0<x<1, \quad t>0, \\
w_{t}(0, t)=-\frac{\eta}{b-a} w_{x}(0, t), \quad t>0 \\
w_{x}(1, t)=\alpha(b-a) w_{t}(1, t)-\frac{\beta(b-a)}{a^{2}} \\
w_{t}^{3}(1, t)-\left(k_{1}+\frac{1}{a}\right)(b-a) w(1, t), t>0 \\
w(x, 0)=w_{0}(b-(b-a) x), \\
w_{t}(x, 0)=w_{1}(b-(b-a) x), \quad 0<x<1
\end{array}\right.
$$

infinitesimal surface element on $\partial \Omega$. Again, from (1.13), we see that the boundary condition on the inner shell $|\boldsymbol{x}|=a$ is self-regulating of the van der Pol type, while that on the outer shell $|\boldsymbol{x}|=b$ injects energy into the system. Because (1.9)-(1.11) are independent of the angular variables (in the spherical coordinate system), the initial-boundary value problem (1.8)-(1.11) has rotational symmetry. So let us

attempt the reduction of dimensionality by writing

Substitution of (1.14) into (1.8)-(1.11) leads to the ollowing initial-boundary value problem:

$$
\begin{aligned}
& \frac{\partial^{2} w(r, t)}{\partial r^{2}}-\frac{1}{c^{2}} \frac{\partial^{2} w(r, t)}{\partial t^{2}}=0, \quad a<r<b, \quad t>0, \\
& -w_{r}(a, t)=\alpha w_{t}(a, t)-\frac{\beta}{a^{2}} w_{t}^{3}(a, t) \\
& \quad-\left(k_{1}+\frac{1}{a}\right) w(a, t), \\
& w_{t}(b, t)=\eta w_{r}(b, t)+\eta\left(k_{2}-\frac{1}{b}\right) w(b, t) \\
& w(r, 0)=w_{0}(r) \equiv W_{0}(r) / r, \\
& w_{t}(r, 0)=w_{1}(r)=W_{1}(r) / r, \quad a<r<b .
\end{aligned}
$$

In the above, $d \boldsymbol{x}=d x_{1} d x_{2} d x_{2}$ and $d \sigma$ are, respectively, the infinitesimal volume element on $\Omega$ and the

Further, setting

$$
\begin{aligned}
& b-a=c, \quad \frac{\eta}{b-a}=\tilde{\eta}, \quad \alpha(b-a)=\tilde{\alpha}, \\
& \frac{\beta(b-a)}{a^{2}}=\tilde{\beta}, \quad\left(k_{1}+\frac{1}{a}\right)(b-a)=\tilde{\gamma},
\end{aligned}
$$

Then (1.17) is converted exactly to the form of (1.1)(1.4). 
Note that, even though we are able to eliminate the $w(b, t)$ term in $(1.15)_{3}$ by $(1.16)$, we cannot eliminate the $w(a, t)$ term in $(1.15)_{2}$ at the same time because its coefficient, $-\left(k_{1}+\frac{1}{a}\right)$, is always negative. $\square$

The statements we have made in Remark 1.1 (2) above actually opens the door for the investigation of chaotic vibration of the wave equation on a multidimensional domain.

The presence of the $\gamma w(1, t)$ term in the boundary condition (1.3) has added significant technical difficulty to the study of chaos for the system (1.1)(1.4). Most of us will agree that there is not yet available a universally accepted definition of chaos for time-dependent partial differential equations. In the case when $\gamma=0$ in (1.3), using the method of characteristics for hyperbolic systems one can extract clearly defined interval maps [Chen et al., 1998a-1998c, 2001], [Huang \& Chen, 2001], which come from wave reflection relations totally characterizing the system, and use them as the natural Poincaré section for the system. Since the definition of chaos for interval maps is more or less standard (see, e.g., [Devaney, 1989]), it is thus possible to classify whether the system is chaotic or not when $\gamma=0$. But when $\gamma \neq 0$ in (1.3), fixed interval maps no longer exist. What we have instead is a nonlinear integrodifferential equation with respect to the $t$ variable on the boundary at $x=1$; see (4.4) below. The presence of the integral term signifies a memory effect. Because the integral term tends to cause the drift of the states out of the invariant region, especially when the time horizon is long, this becomes the most technically challenging part of the paper. It has taken us a long time to analyze this complexity and treat it to a desired degree of satisfaction, fruitless until now.

The way we regard that chaos occurs in the system is from the view of unbounded growth of total variations of snapshots developed by us in [Chen et al., 2001], [Huang \& Chen, 2001]. If a system starts out from some initial data (at $t=0)$ whose total variations over the spatial span is finite. As- sume that whatever prescribed forcing term(s) in the boundary data has bounded total variations over the entire time horizon $t: 0<t<\infty$. If the total variations of the snapshots of the state tend to infinity as $t \rightarrow \infty$, this intuitively speaks for the fact that the system becomes more and more oscillatory, without any limitation and, thus, is chaotic. This point of view is summarized in $\S 2$.

In $\S 3$ and 4, we actually prove that the total variations of the snapshots grow unbounded, for a certain set of initial data. In $\S 3$, we first regard the $\gamma w$ term in (1.3) as a prescribed function $\varepsilon f(t)$; see $(3.1)_{3}$. Thus this $\varepsilon f(t)$ becomes a forcing term in an open-loop, nonlinear boundary condition. We then use a perturbation argument and properties of homoclinic orbits to derive the desired unbounded growth of total variations.

In $\S 4$, we then use the equivalence between an open loop system and a closed loop one to prove that total variations of snapshots do go unbounded as $t \rightarrow \infty$, for sufficiently small $\gamma>0$. Graphics for an example are also illustrated.

\section{Chaotic Vibration as Charac- terized by Unbounded Growth of Total Variations of Snapshots}

Let $I$ denote a closed interval $[a, b]$ and let $f: I \rightarrow \mathbb{R}$. We use $V_{I}(f)$ to denote the total variation of the function $f$ over $I$.

Let us first consider the system (1.1)-(1.4), but with $\gamma=0$ in (1.3). Making the transformation

$$
w_{x}=u+v, \quad w_{t}=u-v
$$

we obtain the following first order symmetric hyperbolic system

$$
\begin{aligned}
\frac{\partial}{\partial t}\left[\begin{array}{l}
u(x, t) \\
v(x, t)
\end{array}\right]= & {\left[\begin{array}{cc}
1 & 0 \\
0 & -1
\end{array}\right] \frac{\partial}{\partial x}\left[\begin{array}{l}
u(x, t) \\
v(x, t)
\end{array}\right], } \\
& 0<x<1, \quad t>0,
\end{aligned}
$$


with the left-end boundary condition

$$
v(0, t)=G_{\eta}(u(0, t)) \equiv \frac{1+\eta}{1-\eta} u(0, t), \quad t>0,
$$

and the right-end boundary condition

$$
u(1, t)=F_{\alpha, \beta}(v(1, t)), \quad t>0,
$$

where for given $x \in \mathbb{R}, y=F_{\alpha, \beta}(x)$ is the unique real solution of the cubic equation

$$
\beta(y-x)^{3}+(1-\alpha)(y-x)+2 x=0 .
$$

The initial conditions for $u$ and $v$ are

$$
\left\{\begin{aligned}
u(x, 0)=u_{0}(x) & \equiv \frac{1}{2}\left[w_{0}^{\prime}(x)+w_{1}(x)\right], \\
v(x, 0)=v_{0}(x) & =\frac{1}{2}\left[w_{0}^{\prime}(x)-w_{1}(x)\right], \\
0<x<1 . &
\end{aligned}\right.
$$

Let us fix $\alpha$ and $\beta$ and write $F_{\alpha, \beta}$ briefly as $F$, in case no ambiguity arises. Similarly, we will also write $G_{\eta}$ briefly as $G$. Then the solution $u$ and $v$ of (2.2)(2.6) can be determined completely by the reflection relations $G$ and $F$. The overall system (2.2)-(2.6) is chaotic if the composite reflection relation $G \circ F$ is chaotic (see, e.g., [Devaney, 1989]).

Assume that

$$
0<V_{[0,1]}\left(u_{0}\right)<\infty, \quad 0<V_{[0,1]}\left(v_{0}\right)<\infty .
$$

Then work in [Chen et al., 2001] shows that for a parameter range of $\eta$ when $G_{\eta} \circ F$ is chaotic, there exists a large class of initial conditions $u_{0}(\cdot)$ and $v_{0}(\cdot)$ satisfying (2.7) such that

$$
\lim _{t \rightarrow \infty} V_{[0,1]}(u(\cdot, t))=\infty, \quad \lim _{t \rightarrow \infty} V_{[0,1]}(v(\cdot, t))=\infty .
$$

The proof of (2.8) in [Chen et al., 2001] uses key properties of a chaotic interval map such as the existence of a periodic orbit of period $2^{k} \cdot m$, where $m$ is an odd integer, or the existence of a homoclinic orbit.

At a more elementary level, the property (2.7) for the system (2.2)-(2.6) hinges on the irregular behavior of the iterates of the composite reflection map
$G \circ F$. Even though $G \circ F$ is defined on the entire real line $\mathbb{R}$, it becomes an interval map if we restrict the domain of definition of $G \circ F$ to an invariant interval; see [Chen et al., 1998a-1998c] or Lemma 3.2 below. For an interval map $f$, the relationship between the chaotic behavior and the property of unbounded growth of total variations of iterates $f^{n}$ of $f$ can be seen in the following two theorems.

Theorem 2.1 ([Huang \& Chen, 2001]). Let I be a finite closed interval of $\mathbb{R}$ and let $f: I \rightarrow I$ be continuous. Assume that $f$ has sensitive dependence on initial data on I [Devaney, 1989]. Then $\lim _{n \rightarrow \infty} V_{J}\left(f^{n}\right)=\infty$ for every closed subinterval $J$ of I. The converse is also true if $f$ has finitely many extremal points.

Theorem 2.2 ([Huang \& Chen, 2001]). Let I be a finite closed interval of $\mathbb{R}$ and let $f: I \rightarrow I$ be continuous with finitely many extremal points. Assume that $\lim _{n \rightarrow \infty} V_{J}\left(f^{n}\right)=\infty$ for every closed subinterval $J$ of $I$. Then the map $f$ has periodic points of prime period $2^{k}$ for $k=1,2,3, \ldots$.

More recently, [Juang \& Shieh, 2001] have shown that under the same conditions as in Theorem 2.2, $f$ actually has a periodic point with prime period $m \cdot 2^{k}$ for some integers $m$ and $k$, where $m$ is odd.

Having rationalized the background of how (2.7) and (2.8) may be related to chaos, we proceed to establish them for the system (1.1)-(1.4) when $\gamma$ in (1.3) lies in a certain range.

\section{An Open-Loop Perturbation Approach to Establish the Un- bounded Growth of Total Vari- ations of Snapshots}

In this section, we will view the $\gamma w(1, t)$ term in (1.3) as an explicitly given perturbation term $\varepsilon f(t)$, for some bounded continuous function $f$, for some small $\varepsilon \in \mathbb{R}$. Thus, even though the $\gamma w(1, t)$ term in 
(1.3) constitutes part of the feedback boundary condition, the substitute term $\varepsilon f(t)$ becomes a forcing term and the new boundary condition at $x=1$ is no longer wholly closed-loop. We consider the model

$$
\left\{\begin{array}{l}
w_{x x}(x, t)-w_{t t}(x, t)=0 \\
0<x<1, \quad t>0 \\
w_{x}(0, t)=-\eta w_{t}(0, t) \\
\eta>0, \quad \eta \neq 1, \quad t>0 \\
w_{x}(1, t)=\alpha w_{t}(1, t)-\beta w_{t}^{3}(1, t)+\varepsilon f(t) \\
0<\alpha<1, \quad \beta>0 \\
w(x, 0)=w_{0}(x), \quad w_{t}(x, 0)=w_{1}(x) \\
0<x<1
\end{array}\right.
$$

Using (2.1) in (3.1) 3 , we obtain

$$
\begin{aligned}
\beta[u(1, t)-v(1, t)]^{3} & +(1-\alpha)[u(1, t)-v(1, t)] \\
& +2 v(1, t)-\varepsilon f(t)=0 .
\end{aligned}
$$

For each given value of $v(1, t)$ and that of $f(t)$, there exists a unique solution $u(1, t)$ of $(3.2)$. We denote the correspondence by

$$
u(1, t)=\bar{F}(v(1, t))
$$

(For the unperturbed case $\varepsilon f(t) \equiv 0$, we retain our old notation $u(1, t)=F(v(1, t))$ as in (2.4).) Further, for $t \in[0,1]$, write

$$
\begin{aligned}
& f_{(i)}(t) \equiv f(i+t), \\
& v_{(i)}(t) \equiv v(i+t) .
\end{aligned}
$$

Throughout the rest of the discussion, we assume that

$f \in B C[0, \infty) \quad(B C(I)$, where $I=[0, \infty)$, is the space of all bounded continuous functions on an interval $I$ ), $|f(t)| \leq M_{0}$, for some $M_{0}>0$, for all $t \in[0, \infty)$.

Henceforth, let us abbreviate $u(1, t)$ and $v(1, t)$ simply as $u(t)$ and $v(t)$, respectively, in case no ambiguity arises. For given $v_{(i)}(t)$ and $\varepsilon f_{(i)}(t), t \in[0,1]$,
$\bar{F}(v(k+t))=\bar{F}_{k}(v)(t)$, for $k=0,1,2, \ldots, t \in[0,1]$.

Lemma 3.1. Let $f$ satisfy (3.3). For given $v_{(i)}(t), t \in[0,1]$, any $i=0,1,2, \ldots$,

$$
\left|\bar{F}_{i}(v)(t)-F\left(v_{(i)}(t)\right)\right| \leq \frac{M_{0} \varepsilon}{1-\alpha} .
$$

$i=0,1,2, \ldots$, we denote by $\bar{F}_{i}(v)(t)$ the unique solution of

$$
\begin{aligned}
\beta\left[\bar{F}_{i}(v)(t)-v_{(i)}(t)\right]^{3} & +(1-\alpha)\left[\bar{F}_{i}(v)(t)-v_{(i)}(t)\right] \\
& +2 v_{(i)}(t)-\varepsilon f_{(i)}(t)=0 .
\end{aligned}
$$

It is easy to see that

Proof. Since

$$
\begin{aligned}
\beta\left[\bar{F}_{i}(v)(t)-v_{(i)}(t)\right]^{3} & +(1-\alpha)\left[\bar{F}_{i}(v)(t)-v_{(i)}(t)\right] \\
+ & 2 v_{(i)}(t)-\varepsilon f_{(i)}(t)=0, \\
\beta\left[F\left(v_{(i)}(t)\right)-v_{(i)}(t)\right]^{3} & +(1-\alpha)\left[F_{i}\left(v_{(i)}\right)(t)-v_{(i)}(t)\right] \\
+ & 2 v_{(i)}(t)=0,
\end{aligned}
$$

by subtraction we have

$$
\begin{aligned}
& \left(\bar{F}_{i}(v)(t)-F\left(v_{(i)}(t)\right)\right)\left\{\beta \left[\left(\bar{F}_{i}(v)(t)-v_{(i)}(t)\right)^{2}\right.\right. \\
& \quad+\left(\bar{F}_{i}(v)(t)-v_{(i)}(t)\right)\left(F\left(v_{(i)}\right)(t)-v_{(i)}(t)\right) \\
& \left.\left.\quad+\left(F\left(v_{(i)}(t)\right)-v_{(i)}(t)\right)^{2}\right]+(1-\alpha)\right\}-\varepsilon f_{(i)}(t)=0 .
\end{aligned}
$$

The terms inside $[\cdots]$ above are nonnegative. Thus

$$
\left|\bar{F}_{i}(v)(t)-F\left(v_{(i)}(t)\right)\right| \cdot(1-\alpha) \leq\left|\varepsilon f_{(i)}(t)\right|
$$

and, therefore,

$$
\left|\bar{F}_{i}(v)(t)-F\left(v_{(i)}(t)\right)\right| \leq \frac{M_{0}|\varepsilon|}{1-\alpha} .
$$

The solution to (3.1) can now be written as follows: for $t=2 k+\tau, k=0,1,2, \ldots, 0 \leq \tau<2$ and 
$0 \leq x \leq 1$

$$
\begin{aligned}
& u(x, t)=\left\{\begin{array}{l}
\left(\bar{F}_{k-1} \circ G\right) \circ\left(\bar{F}_{k-2} \circ G\right) \circ \cdots \circ \\
\left(\bar{F}_{0} \circ G\right)\left[u_{0}(x+\tau)\right], \quad \tau \leq 1-x ; \\
G^{-1} \circ\left(G \circ \bar{F}_{k}\right) \circ\left(G \circ \bar{F}_{k-1}\right) \circ \cdots \circ \\
\left(G \circ \bar{F}_{0}\right)\left[v_{0}(2-x-\tau)\right], \\
1-x<\tau \leq 2-x ; \\
\left(\bar{F}_{k} \circ G\right) \circ\left(\bar{F}_{k-1} \circ G\right) \circ \cdots \circ \\
\left(\bar{F}_{0} \circ G\right)\left[u_{0}(\tau+x-2)\right], \\
2-x<\tau \leq 2 ;
\end{array}\right. \\
& v(x, t)=\left\{\begin{array}{l}
\left(G \circ \bar{F}_{k-1}\right) \circ\left(G \circ \bar{F}_{k-1}\right) \circ \cdots \circ \\
\left(G \circ \bar{F}_{0}\right)\left[v_{0}(x-\tau)\right], \quad \tau \leq x ; \\
G \circ\left(\bar{F}_{k-1} \circ G\right) \circ\left(\bar{F}_{k-2} \circ G\right) \circ \cdots \circ \\
\left(\bar{F}_{0} \circ G\right)\left[u_{0}(\tau-x)\right], \\
\quad x<\tau \leq 1+x ; \\
\left(G \circ \bar{F}_{k}\right) \circ\left(G \circ \bar{F}_{k-1}\right) \circ \cdots \circ \\
\left(G \circ \bar{F}_{0}\right)\left[v_{0}(2+x-\tau)\right], \\
\quad 1+x<\tau \leq 2 .
\end{array}\right.
\end{aligned}
$$

From (3.6) and (3.7), we see that if

$$
\lim _{k \rightarrow \infty} V_{[0,1]}\left(\left(\bar{F}_{k} \circ G\right) \circ \cdots \circ\left(\bar{F}_{0} \circ G\right)\left(u_{0}\right)\right)=\infty,
$$

then we have

$$
\lim _{t \rightarrow \infty} V_{[0,1]}(u(\cdot, t))=\infty, \quad \lim _{t \rightarrow \infty} V_{[0,1]}(v(\cdot, t))=\infty .
$$

Lemma 3.2. Let $\eta>0$ satisfy either

(i) $0<\underline{\eta}_{H} \equiv\left(1-\frac{1+\alpha}{3 \sqrt{3}}\right)\left(1+\frac{1+\alpha}{3 \sqrt{3}}\right)^{-1}$

$$
<\eta<\underline{\eta}_{0}<1,
$$

(ii) $1<\bar{\eta}_{0}<\eta<\bar{\eta}_{H} \equiv\left(1-\frac{1+\alpha}{3 \sqrt{3}}\right)^{-1}$.

$$
\left(1+\frac{1+\alpha}{3 \sqrt{3}}\right)
$$

where $\underline{\eta}_{0}: 0<\underline{\eta}_{0}<1$ and $\bar{\eta}_{0}: 1<\bar{\eta}_{0}<\infty$ are the unique solution of, respectively, the following equations

$$
\begin{gathered}
\frac{1+\underline{\eta}_{0}}{1-\underline{\eta}_{0}} \frac{1+\alpha}{3} \sqrt{\frac{1+\alpha}{3 \beta}}=\frac{1+\underline{\eta}_{0}}{2 \underline{\eta}_{0}} \sqrt{\frac{1+\alpha \underline{\eta}_{0}}{\beta \underline{\eta}_{0}}}, \\
\frac{\bar{\eta}_{0}+1}{\bar{\eta}_{0}-1} \frac{1+\alpha}{3} \sqrt{\frac{1+\alpha}{3 \beta}}=\frac{1+\bar{\eta}_{0}}{2} \sqrt{\frac{\alpha+\bar{\eta}_{0}}{\beta}} .
\end{gathered}
$$

Then

$$
\begin{aligned}
M_{1} & \equiv \text { local maximum of } G_{\eta} \circ F \\
& =\left|\frac{1+\eta}{1-\eta}\right| \frac{1+\alpha}{3} \sqrt{\frac{1+\alpha}{3 \beta}}<\frac{1+\eta}{2} \sqrt{\frac{\alpha+\eta}{\beta}} \\
& \equiv B_{1},
\end{aligned}
$$

and $\left[-B_{1}, B_{1}\right]$ is an invariant interval of $G_{\eta} \circ F$ such that

$$
\begin{array}{ll}
G_{\eta} \circ F\left(M_{1}\right)>0 & \text { for }(3.8), \\
G_{\eta} \circ F\left(M_{1}\right)<0 & \text { for }(3.9) .
\end{array}
$$

Proof. See [Chen et al., 1998b, Lemmas 2.4, 2.5, and Theorems 4.1, 4.2].

We wish to emphasize here that for the parameter ranges of $\eta$ given in (3.8) and (3.9), the map $G_{\eta} \circ F$ has homoclinic orbits in $\left[-B_{1}, B_{1}\right]$ for each such $\eta$. This homoclinic property is crucial for the perturbation arguments in the subsequent sections.

Lemma 3.3. Let $\eta>0$ satisfy either (3.8) or (3.9). Then

(i) for $\eta$ satisfying (3.8),

$$
\begin{aligned}
M_{2} & \equiv \text { local maximum of } F \circ G_{\eta} \\
& =\frac{1+\alpha}{3} \sqrt{\frac{1+\alpha}{3 \beta}}<\frac{1-\eta}{2 \eta} \sqrt{\frac{1+\alpha \eta}{\beta \eta}} \\
& \equiv B_{2}
\end{aligned}
$$

(ii) for $\eta$ satisfying (3.9),

$$
\begin{aligned}
M_{2} & \equiv \text { local maximum of } F \circ G_{\eta} \\
& =\frac{1+\alpha}{3} \sqrt{\frac{1+\alpha}{3 \beta}}<\frac{\eta-1}{2} \sqrt{\frac{\alpha+\eta}{\beta}} \\
& \equiv B_{2},
\end{aligned}
$$

and $\left[-B_{2}, B_{2}\right]$ is an invariant interval of $F \circ G_{\eta}$ such that

$$
\begin{array}{ll}
F \circ G_{\eta}\left(M_{2}\right)>0 & \text { for (3.8), } \\
F \circ G_{\eta}\left(M_{2}\right)<0 & \text { for (3.9). }
\end{array}
$$

Proof. Same as that for Lemma 3.2. 


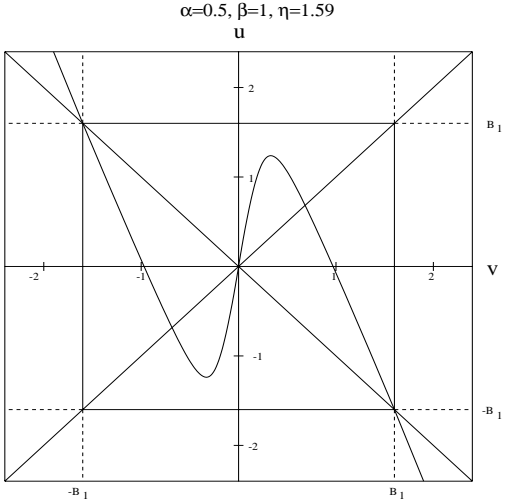

(a)

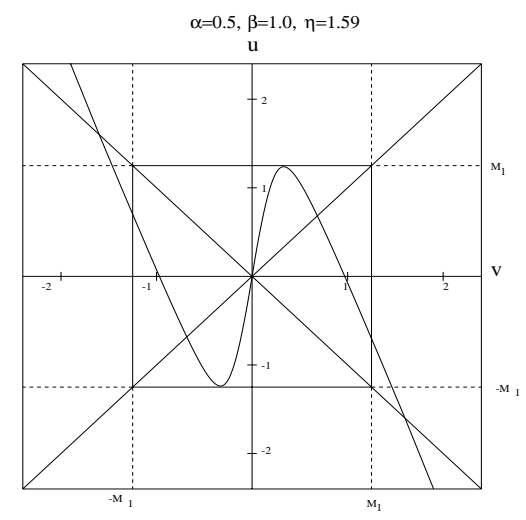

(b)

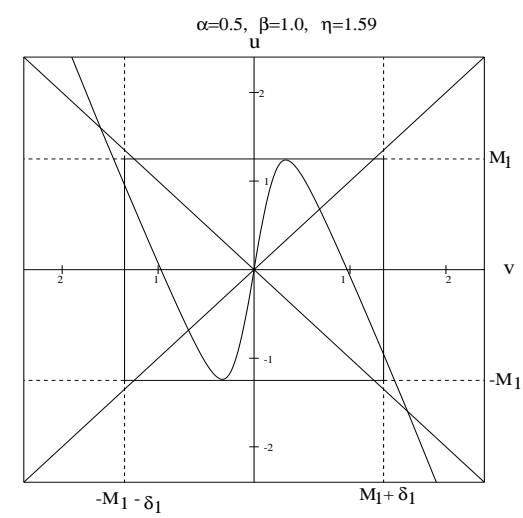

(c)

Figure 3.1: We plot the graph of $G_{\eta} \circ F_{\alpha, \beta}$, with $\alpha=1 / 2, \beta=1$ and $\eta=1.59$. See Remark 3.1. In (a), the square is $\mathcal{S}_{1}$. In (b), the square is $\mathcal{S}_{2}$. In (c), the rectangle is $\mathcal{R}$. The map $G_{\eta} \circ F_{\alpha, \beta}$ is invariant on $\mathcal{S}_{1}, \mathcal{S}_{2}$ and $\mathcal{R}$. Thus, we see the extra margin property from $\mathcal{R}$.

From Lemmas 3.2 and 3.3, we can further choose $\eta$ satisfying (3.8) or (3.9) such that

$$
\begin{aligned}
& G_{\eta} \circ F\left(M_{1}\right)<M_{1}, \\
& F \circ G_{\eta}\left(M_{2}\right)<M_{2}, \quad \text { for }(3.8), \\
& G_{\eta} \circ F\left(M_{1}\right)>-M_{1}, \\
& F \circ G_{\eta}\left(M_{2}\right)>-M_{2}, \quad \text { for }(3.9) .
\end{aligned}
$$

Thus, there exist $\delta_{1}>0$ and $\delta_{2}>0$ such that

$$
\begin{aligned}
& \left(G_{\eta} \circ F\right)\left[-M_{1}-\delta_{1}, M_{1}+\delta_{1}\right] \subseteq\left[-M_{1}, M_{1}\right], \\
& \left(F \circ G_{\eta}\right)\left[-M_{2}-\delta_{2}, M_{2}+\delta_{2}\right] \subseteq\left[-M_{2}, M_{2}\right] .
\end{aligned}
$$

Remark 3.1. Lemmas 3.2 and 3.3 allow us to add a perturbation term in the recursive iterations. Let us explain their significance through Fig. 3.1, where by choosing $\alpha=0.5, \beta=1$ and $\eta=1.59$ and by setting $\delta_{1}$ to satisfy $G_{\eta} \circ F\left(M_{1}+\delta_{1}\right)=M_{1}$ we see the following:

(i) $\mathcal{S}_{1} \equiv\left[-B_{1}, B_{1}\right] \times\left[-B_{1}, B_{1}\right]$ is the large invariant square for the map $G_{\eta} \circ F_{\alpha, \beta}$; see Fig. 3.1 (a);

(ii) $\mathcal{S}_{2} \equiv\left[-M_{1}, M_{1}\right] \times\left[-M_{1}, M_{1}\right]$ is the small invariant square for the map $G_{\eta} \circ F_{\alpha, \beta}$; see Fig. 3.1 (b);

(iii) $\mathcal{R} \equiv\left[-\left(M_{1}+\delta_{1}\right), M_{1}+\delta_{1}\right] \times\left[-M_{1}, M_{1}\right]$ is an invariant rectangle for the map $G_{\eta} \circ F_{\alpha, \beta}$; see Fig. 3.1 (c).

The invariant rectangle in (iii) has width $2\left(M_{1}+\delta_{1}\right)$, which is larger than the height $2 M_{1}$ by a margin $2 \delta_{1}>0$. This extra margin $\delta_{1}$ is crucial, providing what we need in order to allow small perturbations. We call this the extra margin property.

Lemma 3.4. Assume that $\eta$ satisfies Lemma 3.3 and (3.18), and that $\delta_{2}$ satisfies (3.20). Choose $\varepsilon$ such that $|\varepsilon|<\frac{(1-\alpha) \delta_{2}}{M_{0}}$. Then

$$
\begin{aligned}
& \left|\left(\bar{F}_{k} \circ G\right) \circ\left(\bar{F}_{k-1} \circ G\right) \circ \cdots \circ\left(\bar{F}_{0} \circ G\right)\left(u_{0}\right)(t)\right| \\
& \quad \leq M_{2}+\delta_{2}, \quad t \in[0,1]
\end{aligned}
$$

provided that $\left|u_{0}(t)\right| \leq M_{2}+\delta_{2}$ for $t \in[0,1]$, for $k=0,1,2, \ldots$. 
Proof. By Lemma 3.1, we have

$$
\left|\bar{F}_{0} \circ G\left(u_{0}\right)(\tau)-F \circ G\left(u_{0}(\tau)\right)\right| \leq \frac{M_{0}|\varepsilon|}{1-\alpha} .
$$

Therefore

$$
\begin{aligned}
\left|\bar{F}_{0} \circ G\left(u_{0}\right)(\tau)\right| \leq & \left|F \circ G\left(u_{0}(\tau)\right)\right| \\
& +\frac{M_{0}}{1-\alpha} \frac{1-\alpha}{M_{0}} \delta_{2} \\
\leq & M_{2}+\delta_{2}
\end{aligned}
$$

because for $\left|u_{0}(\tau)\right| \leq M_{2}+\delta_{2},\left|F \circ G\left(u_{0}(\tau)\right)\right| \leq M_{2}$.

Inductively, if

$$
\begin{aligned}
& \left|\left(\bar{F}_{k} \circ G\right) \circ\left(\bar{F}_{k-1} \circ G\right) \circ \cdots \circ\left(\bar{F}_{0} \circ G\right)\left(u_{0}\right)(\tau)\right| \\
& \quad \leq M_{2}+\delta_{2}
\end{aligned}
$$

then

$$
\begin{aligned}
& \mid\left(\bar{F}_{k+1} \circ G\right) \circ\left(\bar{F}_{k} \circ G\right) \circ \cdots \circ\left(\bar{F}_{0} \circ G\right)\left(u_{0}\right)(\tau) \\
& \quad-(F \circ G) \circ\left(\bar{F}_{k} \circ G\right) \circ \cdots \circ\left(\bar{F}_{0} \circ G\right)\left(u_{0}\right)(\tau) \mid \\
& \leq \frac{M_{0}}{1-\alpha} \frac{1-\alpha}{M_{0}} \delta_{2}=\delta_{2} .
\end{aligned}
$$

Because $\left|\left(\bar{F}_{k} \circ G\right) \circ\left(\bar{F}_{k-1} \circ G\right) \circ \cdots \circ\left(\bar{F}_{0} \circ G\right)\left(u_{0}\right)(\tau)\right| \leq$ $M_{2}+\delta_{2}$, using the same argument as in (3.21) we have

$$
\begin{aligned}
& \left|\left(\bar{F}_{k+1} \circ G\right) \circ\left(\bar{F}_{k} \circ G\right) \circ \cdots \circ\left(\bar{F}_{0} \circ G\right)\left(u_{0}\right)(\tau)\right| \\
& \quad \leq M_{2}+\delta_{2} .
\end{aligned}
$$

Similarly, we can prove the following.

Lemma 3.5. Assume that $\eta$ satisfies Lemma 3.2 and (3.17), and that $\delta_{1}$ satisfies (3.19). Choose $\varepsilon$ such that $|\varepsilon|<\left|\frac{1-\eta}{1+\eta}\right| \frac{(1-\alpha) \delta_{1}}{M_{0}}$. Then

$$
\begin{aligned}
& \left|\left(G \circ \bar{F}_{k}\right) \circ\left(G \circ \bar{F}_{k-1}\right) \circ \cdots \circ\left(G \circ \bar{F}_{0}\right)\left(v_{0}\right)(t)\right| \\
& \quad \leq M_{1}+\delta_{1}, \quad t \in[0,1],
\end{aligned}
$$

provided that $\left|v_{0}(t)\right| \leq M_{1}+\delta_{1}$ for $t \in[0,1]$, for $k=0,1,2, \ldots$.
Lemma 3.6. Assume the conditions of Lemma 3.3 and (3.16). Let $v_{c}=\left|\frac{1-\eta}{1+\eta}\right| \frac{2-\alpha}{3} \sqrt{\frac{1+\alpha}{3 \beta}}$ be a critical point such that $\left|F \circ G\left(v_{c}\right)\right|=M_{2}$. Then there exists $a \delta_{2}^{\prime}>0$ sufficiently small such that

$$
0<(F \circ G)^{\prime}(v)<1, \quad \text { for } \quad v \in\left[v_{c}-\delta_{2}^{\prime}, v_{c}\right]
$$

and

$$
(F \circ G)\left(M_{2}-2 x\right)+2 x<0, \quad \text { for } \quad 0 \leq x \leq \delta_{2}^{\prime} .
$$

Proof. This follows easily from (3.16) by a continuity argument.

Similarly, we can prove the following.

Lemma 3.7. Assume the conditions of Lemma 3.3 and (3.15). Let $v_{c}=\left|\frac{1-\eta}{1+\alpha}\right| \frac{2-\alpha}{3} \sqrt{\frac{1+\alpha}{3 \beta}}$ be a critical point such that $\left|F \circ G\left(v_{c}\right)\right|=M_{2}$. Then there exists a $\delta_{2}^{\prime \prime}>0$ sufficiently small such that

$$
-1<(F \circ G)^{\prime}(v)<0, \quad \text { for } \quad v \in\left[v_{c}-\delta_{2}^{\prime \prime}, v_{c}\right]
$$

and

$$
(F \circ G)\left(M_{2}-2 x\right)+2 x>0, \quad \text { for } \quad 0 \leq x \leq \delta_{2}^{\prime \prime} .
$$

Lemmas 3.6 and 3.7 deal with the map $F \circ G$. For the map $G \circ F$, the following can be proved in a similar way.

Lemma 3.8. Assume the conditions of Lemma 3.2 and (3.11) or (3.12). Let $v_{c}=\frac{2-\alpha}{3} \sqrt{\frac{1+\alpha}{3 \beta}}$ be a critical point such that $\left|G \circ F\left(v_{c}\right)\right|=M_{1}$. Then (i) for (3.11), there exists a $\delta_{1}^{\prime}>0$ sufficiently small such that

$$
-1<(G \circ F)^{\prime}(v)<0, \quad \text { for } \quad v \in\left[v_{c}-\delta_{1}^{\prime}, v_{c}\right]
$$

and

$$
(G \circ F)\left(M_{1}-2 x\right)+2 x>0, \quad \text { for } \quad 0 \leq x \leq \delta_{1}^{\prime} ;
$$


(ii) for (3.12), there exists a $\delta_{1}^{\prime \prime}>0$ sufficiently Then small such that

$$
0<(G \circ F)^{\prime}(v)<1, \quad \text { for } \quad v \in\left[v_{c}-\delta_{1}^{\prime \prime}, v_{c}\right]
$$

and

$$
\begin{aligned}
& \left|\mathfrak{g}_{0}\left(t_{1}\right)-(F \circ G)\left(u_{0}\left(t_{1}\right)\right)\right| \leq \frac{M_{0} \varepsilon}{1-\alpha}<\tilde{\delta}_{2}, \\
& \left|\mathfrak{g}_{0}\left(t_{1}\right)-(F \circ G)\left(x_{1}\right)\right|=\left|\mathfrak{g}_{0}\left(t_{1}\right)-M_{2}\right|<\tilde{\delta}_{2}, \\
\therefore \quad & \mathfrak{g}_{0}\left(t_{1}\right)>M_{2}-\tilde{\delta}_{2} .
\end{aligned}
$$

$(G \circ F)\left(M_{1}-2 x\right)+2 x<0, \quad$ for $\quad 0 \leq x \leq \delta_{1}^{\prime \prime}$.

Theorem 3.1. Let $\eta$ satisfy the assumptions in Lemma 3.4, and let

$$
|\varepsilon|<\frac{1-\alpha}{M_{0}} \tilde{\delta}_{2}, \quad \tilde{\delta}_{2} \equiv \min \left(\delta_{2}, \delta_{2}^{\prime}\right)
$$

where $\delta_{2}$ and $\delta_{2}^{\prime}$ satisfy (3.20), (3.22) and (3.23). Assume that

$$
\begin{aligned}
& \left|u_{0}(t)\right| \leq M_{2}, \quad 0 \leq t \leq 1 ; \\
& \text { Range } u_{0} \supseteq\left[-\tilde{\delta}_{2}, M_{2}-\tilde{\delta}_{2}\right] .
\end{aligned}
$$

Then

$$
\begin{gathered}
\lim _{k \rightarrow \infty} V_{[0,1]}\left(\left(\bar{F}_{k} \circ G\right) \circ\left(\bar{F}_{k-1} \circ G\right)\right. \\
\left.\circ \cdots \circ\left(\bar{F}_{0} \circ G\right)\left(u_{0}\right)\right)=\infty .
\end{gathered}
$$

Proof. To simplify notation, write

$$
\begin{aligned}
& \mathfrak{g}_{k}(\tau)=\left(\bar{F}_{k} \circ G\right) \circ\left(\bar{F}_{k-1} \circ G\right) \circ \cdots \circ \\
& \quad\left(\bar{F}_{0} \circ G\right)\left(u_{0}\right)(\tau), \quad \tau \in[0,1], \quad k=0,1,2, \ldots .
\end{aligned}
$$

We wish to show

$$
\lim _{k \rightarrow \infty} V_{[0,1]}\left(\mathfrak{g}_{k}\right)=\infty
$$

Let $x_{1} \in\left(0, v_{c}\right)$ satisfy $x_{1}=(F \circ G)^{-1}\left(M_{2}\right)$. Let $t_{0}, t_{1}, t_{2} \in[0,1]$ be such that

$$
u_{0}\left(t_{0}\right)=-\tilde{\delta}_{2}, \quad u_{0}\left(t_{1}\right)=x_{1}, \quad u_{0}\left(t_{2}\right)=M_{2}-\tilde{\delta}_{2} .
$$

We can choose $t_{1}$ such that

$$
\text { either } t_{0}<t_{1}<t_{2} \text {, or } t_{2}<t_{1}<t_{0} \text {. }
$$

Define

$J_{1}=$ the closed interval with endpoints $t_{0}$ and $t_{1}$, $J_{2}=$ the closed interval with endpoints $t_{1}$ and $t_{2}$.
Also

$$
\begin{aligned}
& \left|\mathfrak{g}_{0}\left(t_{2}\right)-(F \circ G)\left(u_{0}\left(t_{2}\right)\right)\right| \\
= & \left|\mathfrak{g}_{0}\left(t_{2}\right)-(F \circ G)\left(M_{2}-\tilde{\delta}_{2}\right)\right| \leq \tilde{\delta}_{2}, \\
\therefore \quad & \mathfrak{g}_{0}\left(t_{2}\right) \leq(F \circ G)\left(M_{2}-\tilde{\delta}_{2}\right)+\tilde{\delta}_{2} .
\end{aligned}
$$

By (3.23) and the fact that $F \circ G$ is decreasing on $\left[v_{c}, M_{2}\right]$, we have

$$
F \circ G\left(M_{2}-\tilde{\delta}_{2}\right)<F \circ G\left(M_{2}-2 \tilde{\delta}_{2}\right) \leq-2 \tilde{\delta}_{2} .
$$

Combining (3.30) and (3.31), we have

$$
\mathfrak{g}_{0}\left(t_{2}\right) \leq F \circ G\left(M_{2}-2 \tilde{\delta}_{2}\right)+\tilde{\delta}_{2} \leq-2 \tilde{\delta}_{2}+\tilde{\delta}_{2}=-\tilde{\delta}_{2} .
$$

Also

$$
\begin{aligned}
& \left|\mathfrak{g}_{0}\left(t_{0}\right)-(F \circ G)\left(u_{0}\left(t_{0}\right)\right)\right| \\
= & \left|\mathfrak{g}_{0}\left(t_{0}\right)-F \circ G\left(-\tilde{\delta}_{2}\right)\right|<\tilde{\delta}_{2}, \\
\therefore \quad & \mathfrak{g}_{0}\left(t_{0}\right)<F \circ G\left(-\tilde{\delta}_{2}\right)+\tilde{\delta}_{2}<-\tilde{\delta}_{2}+\tilde{\delta}_{2}=0, \\
& \quad \text { because }(F \circ G)^{\prime}(0)>1 .
\end{aligned}
$$

From (3.29)-(3.32), we obtain

$$
\begin{aligned}
& \mathfrak{g}_{0}\left(J_{2}\right) \supseteq\left[-\tilde{\delta}_{2}, M_{2}-\tilde{\delta}_{2}\right], \\
& \mathfrak{g}_{0}\left(J_{1}\right) \supseteq\left[0, M_{2}-\tilde{\delta}_{2}\right] .
\end{aligned}
$$

Using the same ideas as in the above, we can show that $J_{2}$ has two subintervals $J_{2,1}$ and $J_{2,2}$, such that

$$
\begin{aligned}
& \mathfrak{g}_{1}\left(J_{2,1}\right)=\bar{F}_{1} \circ G\left(\mathfrak{g}_{0}\left(J_{2,1}\right)\right) \supseteq\left[0, M_{2}-\tilde{\delta}_{2}\right], \\
& \mathfrak{g}_{1}\left(J_{2,2}\right)=\bar{F}_{1} \circ G\left(\mathfrak{g}_{0}\left(J_{2,2}\right)\right) \supseteq\left[-\tilde{\delta}_{2}, M_{2}-\tilde{\delta}_{2}\right] .
\end{aligned}
$$

On the other hand for $J_{1}$, we now show that $J_{1}$ has a subinterval $J_{1,2}$ such that

$$
\mathfrak{g}_{1}\left(J_{1,2}\right) \supseteq\left[-\tilde{\delta}_{2}, M_{2}-\tilde{\delta}_{2}\right] .
$$


Since $0<x_{1}<M_{2}-\varepsilon \leq M_{2}-\tilde{\delta}_{2}$, consider $\left[x_{1}, M_{2}-2 \tilde{\delta}_{2}\right] \supseteq \mathfrak{g}_{1}\left(J_{1}\right) . \quad J_{1}$ thus has a subinterval $J_{1,2}$ such that $\left[x_{1}, M_{2}-2 \tilde{\delta}_{2}\right]=\mathfrak{g}_{1}\left(J_{1,2}\right)$. We have

$$
\begin{aligned}
& \left|\bar{F}_{1} \circ G\left(x_{1}\right)-F \circ G\left(x_{1}\right)\right| \\
= & \left|\bar{F}_{1} \circ G\left(x_{1}\right)-M_{2}\right|<\frac{M_{0} \varepsilon}{1-\alpha}, \\
\therefore & \bar{F}_{1} \circ G\left(x_{1}\right) \geq M_{2}-\frac{M_{0} \varepsilon}{1-\alpha} \geq M_{2}-\tilde{\delta}_{2} .
\end{aligned}
$$

From (3.23),

$$
\begin{aligned}
& F \circ G\left(M_{2}-2 \tilde{\delta}_{2}\right)<-2 \tilde{\delta}_{2}, \\
& \quad \bar{F}_{1} \circ G\left(M_{2}-2 \tilde{\delta}_{2}\right)-F \circ G\left(M_{2}-2 \tilde{\delta}_{2}\right) \mid \\
& \quad \leq \frac{M_{0} \varepsilon}{1-\alpha} \leq \tilde{\delta}_{2}, \\
& \quad \bar{F}_{1} \circ G\left(M_{2}-2 \tilde{\delta}_{2}\right) \leq F \circ G\left(M_{2}-2 \tilde{\delta}_{2}\right)+\tilde{\delta}_{2} \\
& \quad \leq-2 \tilde{\delta}_{2}+\tilde{\delta}_{2}=\tilde{\delta}_{2} .
\end{aligned}
$$

Therefore, (3.35) follows from (3.36) and (3.37).

Continuing this argument inductively, we can construct a sequence of subintervals

$$
J_{i_{0}, i_{1}, i_{2} \ldots i_{n}}, \quad i_{k} \in\{1,2\}, \text { for } k=0,1, \ldots, n,
$$

of $J_{i_{0}}$ such that

$$
\begin{aligned}
& \mathfrak{g}_{k}\left(J_{i_{0}, i_{1} i_{2} \ldots i_{k}}\right) \supseteq\left[0, M_{2}-\delta_{2}\right] \quad \text { if } i_{k}=1, \\
& \mathfrak{g}_{k}\left(J_{i_{0}, i_{1} i_{2} \ldots i_{k}}\right) \supseteq\left[-\delta_{2}, M_{2}-\delta_{2}\right] \quad \text { if } i_{k}=2 .
\end{aligned}
$$

Note that in (3.38), an index $i_{j}=1$ can only follow $i_{j-1}=2$; the subscript $i_{0}, i_{1} \ldots i_{k}$ consists of all combinations of $i_{j} \in\{0,1\}$ except those when two adjacent indices $i_{j-1} i_{j}$ are 11 . Therefore, by summing over all such admissible $i_{o}, i_{1} \ldots i_{k}$, we have

$$
\begin{aligned}
V_{[0,1]}\left(\mathfrak{g}_{k}\right) & \geq \sum V_{J_{i_{0}, i_{1} \ldots i_{k}}}\left(\mathfrak{g}_{k}\right) \\
& \geq 2^{k-1}\left(M_{2}-\delta_{2}\right) \rightarrow \infty, \text { as } k \rightarrow \infty,
\end{aligned}
$$

and (3.28) has been proved.

Theorem 3.1 covers just the case under the conditions of Lemma 3.4. If, instead, the conditions are those stated in Lemma 3.5, then a proof can be similarly established that

$$
\begin{aligned}
& \lim _{k \rightarrow \infty} V_{[0,1]}\left(\left(G \circ \bar{F}_{k}\right) \circ\left(G \circ \bar{F}_{k-1}\right) \circ \cdots \circ\right. \\
& \left.\quad\left(G \circ \bar{F}_{0}\right)\left(v_{0}\right)\right)=\infty
\end{aligned}
$$

$$
\left|v_{0}(t)\right| \leq M_{1}, \quad 0 \leq t \leq 1
$$

$$
\text { Range } v_{0} \supseteq\left[-\tilde{\delta}_{1}, M_{1}-\tilde{\delta}_{1}\right]
$$

for

$$
|\varepsilon|<\left|\frac{1+\eta}{1-\eta}\right| \frac{1-\alpha}{M_{0}} \tilde{\delta}_{1}, \quad \tilde{\delta}_{1} \equiv \min \left(\delta_{1}, \delta_{1}^{\prime}\right) .
$$

We omit the details.

\section{Chaotic Vibration in the Sense of Unbounded Growth of Snap- shots for the van der Pol Bound- ary Condition Containing Dis- placement}

We now proceed to study the system (1.1)-(1.4). The focus is the boundary condition (1.3). Write

$$
X(t)=u(t)-v(t)
$$

Then

$$
\begin{aligned}
w(1, t) & =\int_{0}^{t} w_{t}(1, \tau) d \tau+a_{0} \quad\left(a_{0} \equiv w(1,0)\right) \\
& =\int_{0}^{t} X(\tau) d \tau+a_{0}
\end{aligned}
$$

For each reflection of waves at $x=1$, we need only consider $0 \leq t \leq 1$. Note that because

$$
\begin{aligned}
a_{n} & \equiv w(1, n)=a_{0}+\int_{0}^{n} X(\tau) d \tau \\
& =a_{n-1}+\int_{n-1}^{n} X(\tau) d \tau,
\end{aligned}
$$

if we know $a_{n}, u(1, t), v(1, t)$ for $t \in[n-1, n)$, then we can determine $a_{n+1}, u(1, t), v(1, t)$ for $t \in[n, n+1)$.

Let us now consider the nonlinear wave reflection operator at $x=1$. For the first wave reflection at $x=1$, i.e., for $t \in[0,1]$, using (2.1) and (4.2), we rewrite (1.3) as

$$
\begin{gathered}
\beta X^{3}(t)+(1-\alpha) X(t)+\gamma\left[\int_{0}^{t} X(\tau) d \tau+a_{0}\right] \\
+2 v(t)=0, \quad 0 \leq t \leq 1 .
\end{gathered}
$$


Lemma 4.1. Let $\beta>0,0<\alpha<1, \gamma>0$. Then for any $v \in B C[0,1]$ and $a_{0} \in \mathbb{R}$, the equation (4.4) has a unique solution $X \in B C[0,1]$.

Proof. (i) Uniqueness: Let $a \in \mathbb{R}$ and $v \in B C[0,1]$ be given. Assume that

$$
\begin{aligned}
\beta X_{i}^{3}(t) & +(1-\alpha) X_{i}(t)+\gamma\left[\int_{0}^{t} X_{i}(\tau) d \tau+a\right] \\
& +2 v(t)=0 \text { for } i=1,2 .
\end{aligned}
$$

Then subtraction gives

$$
\begin{aligned}
& \beta Y(t)\left\{\left[X_{1}^{2}(t)+X_{1}(t) X_{2}(t)+X_{2}^{2}(t)\right]+(1-\alpha)\right\} \\
& \quad+\gamma \int_{0}^{t} Y(\tau) d \tau=0 .
\end{aligned}
$$

where $Y \equiv X_{1}-X_{2}$. If $X_{1} \not \equiv X_{2}$ on $[0,1]$, then

$$
\begin{aligned}
P(t) \equiv & \beta\left\{\left[X_{1}^{2}(t)+X_{1}(t) X_{2}(t)+X_{2}^{2}(t)\right]\right. \\
& +(1-\alpha)\}>0 \text { on }[0,1]
\end{aligned}
$$

From (4.5), we have

$$
\beta P(t) Y(t)+\gamma \int_{0}^{t} Y(\tau) d \tau=0, \quad Y(0)=0 .
$$

By a Gronwall argument, we can show that

$$
Y \equiv 0 \text { on }[0,1], \text { a contradiction. }
$$

Therefore, the uniqueness follows.

(ii) Existence and smoothness: Let us construct the solution of (4.4) iteratively as follows. Choose $X_{0}(t)$ to be the (unique) solution of (4.4) when $\gamma=0$. Then $X_{0} \in B C[0,1]$. Iterate by solving $X_{j+1}$ from

$$
\begin{aligned}
\beta X_{j+1}^{3}(t) & +(1-\alpha) X_{j+1}(t) \\
& +\left\{\gamma \int_{0}^{t} X_{j}(\tau) d \tau+2 v(t)\right\}=0 .
\end{aligned}
$$

Then $X_{j+1}$ is unique and $X_{j+1} \in B C[0,1]$. By subtracting $(4.6)_{j=n}$ with $(4.6)_{j=n-1}$, we obtain

$$
\begin{gathered}
{\left[X_{n+1}(t)-X_{n}(t)\right]\left\{\beta \left[X_{n+1}^{2}(t)+X_{n+1}(t) X_{n}(t)\right.\right.} \\
\left.\left.+X_{n}^{2}(t)\right]+(1-\alpha)\right\} \\
+\gamma \int_{0}^{t}\left[X_{n}(\tau)-X_{n-1}(\tau)\right] d \tau=0
\end{gathered}
$$

$$
\begin{aligned}
& X_{n+1}(t)-X_{n}(t)= \\
& -\frac{\gamma}{\beta\left[X_{n+1}^{2}(t)+X_{n+1}(t) X_{n}(t)+X_{n}^{2}(t)\right]+(1-\alpha)} \\
& \quad \cdot \int_{0}^{t}\left[X_{n}(\tau)-X_{n-1}(\tau)\right] d \tau,
\end{aligned}
$$$$
\begin{aligned}
& \left|X_{n+1}(t)-X_{n}(t)\right| \leq \frac{\gamma}{1-\alpha} \int_{0}^{t}\left|X_{n}(\tau)-X_{n-1}(\tau)\right| d \tau \\
& \quad \leq\left(\frac{\gamma}{1-\alpha}\right)^{2} \int_{0}^{t} \int_{0}^{\tau_{1}}\left|X_{n-1}\left(\tau_{2}\right)-X_{n-1}\left(\tau_{2}\right)\right| d \tau_{2} d \tau_{1}
\end{aligned}
$$$$
\leq \cdots
$$$$
\leq\left(\frac{\gamma}{1-\alpha}\right)^{n} \int_{0}^{t} \int_{0}^{\tau_{1}} \int_{0}^{\tau_{2}} \cdots \int_{0}^{\tau_{n-1}}
$$$$
\left|X_{1}\left(\tau_{n}\right)-X_{0}\left(\tau_{n}\right)\right| d \tau_{n} d \tau_{n-1} \ldots d \tau_{2} d \tau_{1}
$$$$
\leq C_{1} \cdot\left(\frac{\gamma}{1-\alpha}\right)^{n} \int_{0}^{t} \int_{0}^{\tau_{1}} \int_{0}^{\tau_{2}} \cdots \int_{0}^{\tau_{n-1}}
$$$$
d \tau_{n} d \tau_{n-1} \ldots d \tau_{2} d \tau_{1}
$$

$$
\begin{aligned}
& \left(C_{1} \equiv \max _{t \in[0,1]}\left|X_{1}(t)-X_{0}(t)\right|\right) \\
= & C_{1} \cdot\left(\frac{\gamma}{1-\alpha}\right)^{n} \frac{t^{n}}{n !} \rightarrow 0 \text { as } n \rightarrow \infty,
\end{aligned}
$$

uniformly on $[0,1]$.

Therefore

$$
\lim _{n \rightarrow \infty} X_{n}=X \in B C[0,1]
$$

for a limit function $X$ satisfying (4.4).

Note that the uniqueness result in Lemma 4.1 guarantees the uniqueness of the solution $w$ for the system (1.1)-(1.4).

The following lemma contains the most needed important information about the boundedness of the $\gamma w(1, t)$ term in (1.3).

Lemma 4.2 (Key technical lemma). Let $w$ be the solution of (1.1)-(1.4). Let $w_{0}$ and $w_{1}$ in (1.4) be sufficiently smooth and are compatible with the boundary conditions (1.2)-(1.3). Assume that $u_{0}$ and $v_{0}$ in (2.6) satisfy

$$
\begin{aligned}
& \left|v_{0}(x)\right| \leq M_{1}, \quad\left|u_{0}(x)\right| \leq M_{2}, \quad x \in[0,1], \\
& \text { (cf. } M_{1} \text { and } M_{2} \text {, respectively, in } \\
& \text { Lemmas 3.2 and 3.3). }
\end{aligned}
$$


Then there exists an $M^{\prime}>0$ (prescribed in (4.22) below) such that if

$$
\left|a_{0}\right| \leq M^{\prime}
$$

then

$$
\left|a_{n}\right| \leq M^{\prime} \quad \text { for } \quad n=1,2, \ldots
$$

and

$$
\begin{array}{ll}
|v(t)| \leq M_{1}, \quad|u(t)| \leq M_{2} & \text { and } \\
|w(1, t)| \leq M^{\prime}+M_{1}+M_{2}, & \forall t \in[0, \infty)
\end{array}
$$

provided that $\gamma>0$ is sufficiently small (satisfying (4.24) below).

Proof. Let us consider the outcome of each reflection at $x=1$. For $t \in[n, n+1]$, from (4.3) and (4.6),

$$
\begin{aligned}
\beta X_{(n)}^{3}(t) & +(1-\alpha) X_{(n)}(t)+2 v_{(n)}(t) \\
& +\gamma\left[\int_{0}^{t} X_{(n)}(\tau) d \tau+a_{n}\right]=0, \quad 0 \leq t \leq 1,
\end{aligned}
$$

where

$$
\begin{aligned}
a_{n} & =w(1, n), u_{(n)}(t)=u(n+t), \\
v_{(n)}(t) & =v(n+t), X_{(n)}(t)=u_{(n)}(t)-v_{(n)}(t) .
\end{aligned}
$$

As in [Chen et al., 1998a, 1998b], for any $v \in \mathbb{R}$, let $g(v)$ be the unique real solution of the cubic equation

$$
\beta g^{3}(v)+(1-\alpha) g(v)+2 v=0 .
$$

The $X_{(n)}(t)$ in (4.9) satisfies, for $0 \leq t \leq 1$,

$$
\begin{aligned}
X_{(n)}(t)= & g\left(v_{(n)}(t)+\frac{\gamma}{2}\left[\int_{0}^{t} X_{(n)}(\tau) d \tau+a_{n}\right]\right) \\
= & g\left(v_{(n)}(t)+\frac{\gamma}{2}\left[\int_{0}^{t} X_{(n)}(\tau) d \tau+a_{n}\right]\right) \\
& -g(0) \quad(\because g(0)=0) \\
= & g^{\prime}(h(t)) \\
& \cdot\left\{v_{(n)}(t)+\frac{\gamma}{2}\left[\int_{0}^{t} X_{(n)}(\tau) d \tau+a_{n}\right]\right\},
\end{aligned}
$$

where $h(t)$, by the Mean Value Theorem, is a continuous function taking values between 0 and $v_{(n)}(t)+$ $\frac{\gamma}{2}\left[\int_{0}^{t} X_{(n)}(\tau) d \tau+a_{n}\right]$. For any $x \in \mathbb{R}$, from [Chen, et al., 1998a, (3.5), p. 4279]

$$
\begin{gathered}
g^{\prime}(x)=-\frac{2}{3 \beta g^{2}(x)+(1-\alpha)}<0, \\
\left|g^{\prime}(x)\right| \leq \frac{2}{1-\alpha}, \quad \lim _{x \rightarrow \pm \infty}\left|g^{\prime}(x)\right|=0 .
\end{gathered}
$$

From (4.11),

$$
\begin{aligned}
X_{(n)}(t) & +p(t) \cdot \gamma\left[\int_{0}^{t} X_{(n)}(\tau) d \tau+a_{n}\right] \\
& =-2 p(t) v_{(n)}(t),
\end{aligned}
$$

where

$$
p(t) \equiv-(1 / 2) g^{\prime}(h(t))>0, \quad p(t) \leq \frac{1}{1-\alpha} .
$$

From (4.12), by Gronwall's method,

$$
\begin{gathered}
\frac{d}{d t}\left\{e^{\gamma \int_{0}^{t} p(\tau) d \tau}\left[\int_{0}^{t} X_{(n)}(\tau) d \tau+a_{n}\right]\right\}= \\
-2 p(t) e^{\gamma \int_{0}^{t} p(\tau) d \tau} v_{(n)}(t) \\
e^{\gamma \int_{0}^{t} p(\tau) d \tau}\left[\int_{0}^{t} X_{(n)} d \tau+a_{n}\right]-a_{n}
\end{gathered}
$$$$
=-2 \int_{0}^{t} p(s) v_{(n)}(s) e^{\gamma \int_{0}^{s} p(\tau) d \tau} d s,
$$$$
\int_{0}^{t} X_{(n)}(\tau) d \tau+a_{n}=
$$$$
a_{n} e^{-\gamma \int_{0}^{t} p(\tau) d \tau}-2 \int_{0}^{t} p(s) v_{(n)}(s) e^{-\gamma \int_{0}^{t} p(\tau) d \tau} d s,
$$$$
\left|\int_{0}^{t} X_{(n)}(\tau) d \tau+a_{n}\right| \leq\left|a_{n}\right| e^{-\gamma \int_{0}^{t} p(\tau) d \tau}+
$$$$
\frac{2}{1-\alpha} \int_{0}^{t}\left|v_{(n)}(s)\right| e^{-\gamma \int_{s}^{t} p(\tau) d \tau} d s .
$$

Assume that

$$
\left|v_{(j)}(t)\right| \leq M_{1}, \quad\left|u_{(j)}(t)\right| \leq M_{2}, \quad\left|a_{j}\right| \leq M^{\prime}
$$

are satisfied, for $j=0,1, \ldots, n$, where $M^{\prime}$ will be determined below in (4.22). Then

$$
\begin{aligned}
& \left|v_{(n)}(t)+\frac{\gamma}{2}\left[\int_{0}^{t} X_{(n)}(\tau) d \tau+a_{n}\right]\right| \\
\leq & \left|v_{(n)}(t)+\frac{\gamma}{2}\left[\int_{0}^{t}\left|X_{(n)}(\tau)\right| d \tau+\left|a_{n}\right|\right]\right| \\
\leq & M_{1}+\frac{\gamma}{2}\left[M_{1}+M_{2}+M^{\prime}\right] \equiv M_{3} .
\end{aligned}
$$


Therefore, by letting

$$
M^{\prime \prime} \equiv \max _{x \in\left[0, M_{3}\right]} g^{2}(x),
$$

we have $M^{\prime \prime}=g^{2}\left(M_{3}\right)$ because $g$ is monotone decreasing, $g(-x)=-g(x)$ and $g(x)<0$ for $x<0$. We have

$$
\begin{aligned}
& \frac{1}{3 \beta M^{\prime \prime}+(1-\alpha)} \leq p(t)=-\frac{1}{2} g^{\prime}(h(t))= \\
& \frac{1}{3 \beta g^{2}(h(t))+(1-\alpha)} \leq \frac{1}{1-\alpha} .
\end{aligned}
$$

From (4.17),

$$
\begin{aligned}
-\frac{1}{1-\alpha}(t-s) & \leq-\int_{s}^{t} p(\tau) d \tau \\
& \leq-\frac{1}{3 \beta M^{\prime \prime}+(1-\alpha)}(t-s) .
\end{aligned}
$$

Hence, from (4.18),

$$
\begin{aligned}
\left|a_{n}\right| e^{-\gamma \int_{0}^{t} p(\tau) d \tau} & \leq\left|a_{n}\right| e^{-\gamma \int_{0}^{t} \frac{1}{3 \beta M^{\prime \prime}+(1-\alpha)} d \tau} \\
& =\left|a_{n}\right| e^{-\frac{\gamma}{3 \beta M^{\prime \prime}+(1-\alpha)} t}
\end{aligned}
$$

and from (4.13), (4.14) and (4.18),

$$
\begin{aligned}
& \frac{2}{1-\alpha} \int_{0}^{t}\left|v_{(n)}(s)\right| e^{-\gamma \int_{s}^{t} p(\tau) d \tau} d s \\
\leq & \frac{2 M_{1}}{1-\alpha} \int_{0}^{t} e^{-\frac{\gamma(t-s)}{3 \beta M^{\prime \prime}+(1-\alpha)}} d s \\
= & \frac{2 M_{1}}{1-\alpha}\left\{\frac{3 \beta M^{\prime \prime}+(1-\alpha)}{\gamma}\left[1-e^{-\frac{\gamma t}{3 \beta M^{\prime \prime}+(1-\alpha)}}\right]\right\} .
\end{aligned}
$$

From (4.13), (4.19) and (4.20),

$$
\begin{gathered}
|w(1, n+1)|=\left|a_{n+1}\right|=\left|\int_{0}^{1} X_{(n)}(\tau) d \tau+a_{n}\right| \\
\leq\left|a_{n}\right| e^{-\frac{\gamma}{3 \beta M^{\prime \prime}+(1-\alpha)}}+\frac{2 M_{1}}{1-\alpha} \\
\cdot\left\{\frac{3 \beta M^{\prime \prime}+(1-\alpha)}{\gamma}\left[1-e^{-\frac{\gamma}{3 \beta M^{\prime \prime}+(1-\alpha)}}\right]\right\} .
\end{gathered}
$$

But

$$
\begin{aligned}
& \frac{3 \beta M^{\prime \prime}+(1-\alpha)}{\gamma}\left[1-e^{-\frac{\gamma}{3 \beta M^{\prime \prime}+(1-\alpha)}}\right] \\
& =1-\frac{1}{2} \frac{\gamma}{3 \beta M^{\prime \prime}+(1-\alpha)} \\
& +\frac{1}{3 !}\left[\frac{\gamma}{3 \beta M^{\prime \prime}+(1-\alpha)}\right]^{2} \pm \cdots \\
& \leq 1-\frac{c_{0} \gamma}{2\left[3 \beta M^{\prime \prime}+(1-\alpha)\right]}
\end{aligned}
$$

for some $c_{0}: 0<c_{0}<1$,

if $0<\gamma<c_{1}$, for some small $c_{1}$.

Therefore, if we choose $M^{\prime}>0$ such that

$$
\begin{aligned}
& M^{\prime} e^{-\frac{\gamma}{3 \beta M^{\prime \prime}+(1-\alpha)}}+\frac{2 M_{1}}{1-\alpha}\left[1-\frac{c_{0} \gamma}{2\left(3 \beta M^{\prime \prime}+(1-\alpha)\right)}\right] \\
& \leq M^{\prime}, \\
& \text { i.e., } \\
& \qquad \begin{aligned}
M^{\prime} \geq & {\left[1-e^{-\frac{\gamma}{3 \beta M^{\prime \prime \prime}+(1-\alpha)}}\right]^{-1} } \\
& \cdot \frac{2 M_{1}}{1-\alpha}\left[1-\frac{c_{0} \gamma}{2\left(3 \beta M^{\prime \prime}+(1-\alpha)\right)}\right],
\end{aligned}
\end{aligned}
$$

then from (4.21)

$$
\left|a_{n+1}\right| \leq M^{\prime}
$$

(Here let us make a little clarification. $M^{\prime \prime}$ is defined in (4.16) depending on $M_{3}$, while $M_{3}$ is defined in (4.15) depending on $M_{1}, M_{2}$ and $M_{1}^{\prime}$. From (4.22), $M^{\prime}$ in turn depends on $M_{1}$ and $M^{\prime \prime}$. Thus it appears that we were having a vicious cycle of $M^{\prime \prime}$ depending on $M^{\prime \prime}$ itself. However, note that in (4.15), $\gamma$ is very small, making $M_{3}$ depend mainly on $M_{1}$. Therefore (4.22) can be satisfied without problem, for small $\gamma>0$.) The above estimate also gives

$$
|w(1, n+t)| \leq M^{\prime}+\left(M_{1}+M_{2}\right) t, \quad t \in[0,1] .
$$

Since for $t \in[0,1]$,

$$
\begin{aligned}
& v(1,2+n+t)= \\
& G_{\eta} F_{\alpha, \beta}\left(v(1, n+t)+\frac{\gamma}{2} w(1, n+t)\right), \\
& u(1,2+n+t)= \\
& F_{\alpha, \beta}\left(G_{\eta}(u(1, n+t))+\frac{\gamma}{2} w(1,1+n+t)\right),
\end{aligned}
$$


if $\gamma$ is sufficiently small such that

$$
\begin{aligned}
\left|\frac{\gamma}{2} w(1, n+t)\right| & \leq \frac{\gamma}{2}\left(M^{\prime}+M_{1}+M_{2}\right) \\
& \leq \tilde{\delta}_{3} \equiv \min \left(\delta_{1}, \delta_{2}\right),
\end{aligned}
$$

then by Lemmas 3.2, 3.3, (3.19), (3.20), we can use the extra margin property in Remark 3.1 (iii) to conclude that

$$
\begin{aligned}
& |v(1, t)| \leq M_{1} \quad|u(1, t)| \leq M_{2}, \\
& \quad \text { for all } t \in[0, \infty) .
\end{aligned}
$$

Finally, we have laid all the ground work for deriving the chaotic property of vibration of the system (1.1)-(1.4).

Theorem 4.1. Consider (1.1)-(1.4). Assume that $M_{1}, M_{2}$ and $M^{\prime}$ satisfy the conditions in Lemma 4.2. Let $w_{0}$ and $w_{1}$ in (1.4) be sufficiently smooth and be compatible with the boundary conditions (1.2)-(1.3) such that $u_{0}=\frac{1}{2}\left(w_{0}^{\prime}+w_{1}\right)$ and $v_{0}=\frac{1}{2}\left(w_{0}^{\prime}-w_{1}\right)$ satisfy

$$
\left|v_{0}(x)\right| \leq M_{1}, \quad\left|u_{0}(x)\right| \leq M_{2}, \quad x \in[0,1] .
$$

Assume that $\gamma>0$ is sufficiently small such that

$$
\begin{aligned}
& \gamma<\frac{1-\alpha}{M^{\prime}+M_{1}+M_{2}} \tilde{\delta}, \quad \tilde{\delta} \equiv \min \left(\tilde{\delta}_{1}, \tilde{\delta}_{2}\right), \\
& \quad c f . \text { (3.26) and (3.41), respectively, } \\
& \text { for } \tilde{\delta}_{1} \text { and } \tilde{\delta}_{2},
\end{aligned}
$$

$$
\begin{aligned}
& \text { Range } v_{0} \supseteq\left[-\tilde{\delta}, M_{1}-\tilde{\delta}\right], \\
& \text { Range } u_{0} \supseteq\left[-\tilde{\delta}, M_{2}-\tilde{\delta}\right] \text {. }
\end{aligned}
$$

Then for $u=\frac{1}{2}\left(w_{x}+w_{t}\right), v=\frac{1}{2}\left(w_{x}-w_{t}\right)$, we have

$$
\left\{\begin{array}{c}
\lim _{t \rightarrow \infty} V_{[0,1]}(u(\cdot, t))=\infty, \\
\lim _{t \rightarrow \infty} V_{[0,1]}(v(\cdot, t))=\infty .
\end{array}\right.
$$

Proof. By Lemma 4.1, the solution $w$ to the system (1.1)-(1.4) is unique. Under the assumptions of Lemma 4.2 , we have $|w(1, t)| \leq M^{\prime}+M_{1}+M_{2}$ for all $t \geq 0$. Therefore, we can denote

$$
f(t) \equiv w(1, t), \quad t \in[0, \infty), \quad \varepsilon \equiv \gamma,
$$

and regard the closed loop system (1.1)-(1.4) as the open loop system (3.1). Just let $M_{0} \equiv M^{\prime}+M_{1}+M_{2}$. Let

$$
\varepsilon=\gamma<\frac{1-\alpha}{M_{0}} \tilde{\delta}, \quad \tilde{\delta}=\min \left(\tilde{\delta}_{1}, \tilde{\delta}_{2}\right)
$$

Then Theorem 3.1 (3.28), and (3.39) are applicable, and we conclude (4.25).

The consequence in (4.25) also implies that

$$
\left\{\begin{array}{c}
\lim _{t \rightarrow \infty} V_{[0,1]}\left(w_{x}(\cdot, t)\right)=\infty, \\
\lim _{t \rightarrow \infty} V_{[0,1]}\left(w_{t}(\cdot, t)\right)=\infty .
\end{array}\right.
$$

Therefore, the gradient $w$ of (1.1)-(1.4) is chaotic in the sense of unbounded growth of total variations of the snapshots. The proof of (4.26) follows from (4.25) by a little extra work utilizing (2.1) so we omit it here.

One may also question whether

$$
\lim _{t \rightarrow \infty} V_{[0,1]}(w(\cdot, t))=\infty
$$

holds under the assumptions of Theorem 4.1. The answer is negative.

Corollary 4.1. Assume that Theorem 4.1 holds. Then

$$
\begin{aligned}
& V_{[0,1]}(w(\cdot, t)) \leq M_{1}+M_{2}, \\
& \quad \text { for all } t>0 .
\end{aligned}
$$

Proof. We know that

$$
\begin{aligned}
w(x, t) & =w(0, t)+\int_{0}^{x} w_{x}(\xi, t) d \xi \\
& =w(0, t)+\int_{0}^{x}[u(\xi, t)+v(\xi, t)] d \xi, \\
& \text { for } 0 \leq x \leq 1 .
\end{aligned}
$$

Therefore

$$
\begin{aligned}
& V_{[0,1]}(w(\cdot, t)) \\
& \quad=\sup _{\mathcal{P}} \sum_{i=0}^{n}\left|w\left(x_{i+1}, t\right)-w\left(x_{i}, t\right)\right|,
\end{aligned}
$$


where $\mathcal{P}$ is an arbitrary partition of $[0,1]: \mathcal{P}=\left\{x_{i} \mid\right.$ $\left.i=0,1, \ldots, n, 0=x_{0}<x_{1}<\cdots<x_{n}=1\right\}$, such that $\max _{0 \leq i \leq n}\left|x_{i+1}-x_{i}\right| \rightarrow 0$ as $n \rightarrow \infty$. Hence

$$
\begin{aligned}
& V_{[0,1]}(w(\cdot, t)) \\
& \quad=\sup _{\mathcal{P}}\left|\sum_{i=0}^{n-1} \int_{i_{i}}^{x_{i+1}}(u(\xi, t)+v(\xi, t)) d \xi\right| \\
& \quad \leq \sup _{\mathcal{P}} \sum_{i=0}^{n-1} \int_{x_{i}}^{x_{i+1}}|u(\xi, t)+v(\xi, t)| d \xi \\
& \quad \leq\left(M_{1}+M_{2}\right) \sup _{\mathcal{P}} \sum_{i=0}^{n-1}\left(x_{i+1}-x_{i}\right) \\
& \quad=M_{1}+M_{2} .
\end{aligned}
$$

Example 4.1. Let us choose

$$
\begin{gathered}
\left.\begin{array}{c}
w_{0}(x)=0.5-0.95 x+\frac{1}{2} x^{2}, \\
w_{1}(x)=-0.95 x-x,
\end{array}\right\} 0 \leq x \leq 1 \\
\alpha=0.5, \beta=1.95, \gamma=0.01,
\end{gathered}
$$

in (1.2)-(1.4). Then

$$
u_{0}(x)=0.05, v_{0}(x)=x-1
$$

in (2.6). We plot the graphics of $u(x, t), v(x, t)$ and $w(x, t)$ for $t=30$ in Fig. 4.1 (a), (b) and (c). The reader may find that the snapshots of $u$ and $v$ display rather chaotic oscillatory behavior, while that of $w$ doesn't.

There are some still unresolved questions:

(a) We believe that for the parameter range of $\eta$ prescribed in Lemmas 3.2 and 3.3, the chaotic property (4.25) holds for a large class of initial data $w_{0}, u_{0}$ and $v_{0}$ without the requirement that $\gamma>0$ be small. But, how do we analyze the case when $\gamma>0$ is not small?

(b) Even if the map $G_{\eta} \circ F_{\alpha, \beta}$ does not have homoclinic orbits when $\eta$ does not belong to the parameter range as prescribed in Lemmas 3.2 and 3.3 (with $\alpha$ and $\beta$ being fixed), as long as

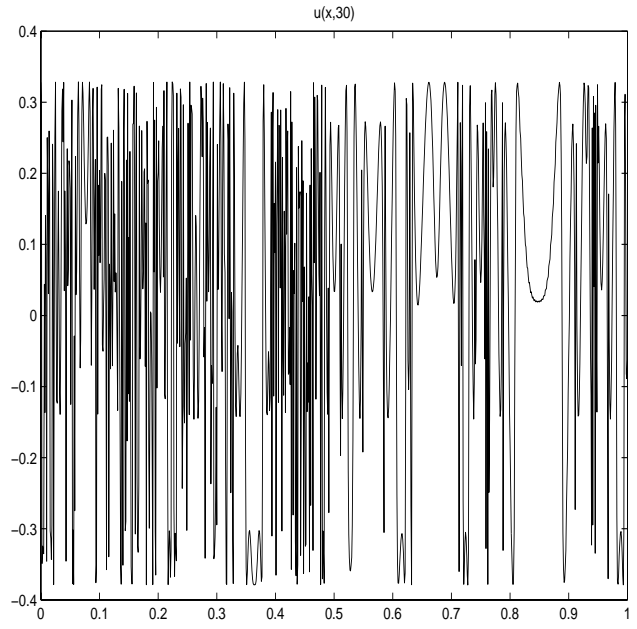

(a) $u(x, t), \quad 0 \leq x \leq 1, \quad t=30$.

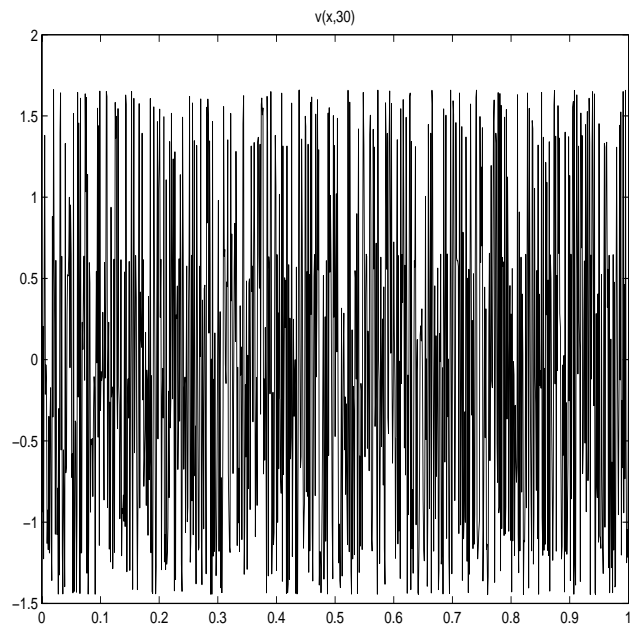

(b) $v(x, t), \quad 0 \leq x \leq 1, \quad t=30$.

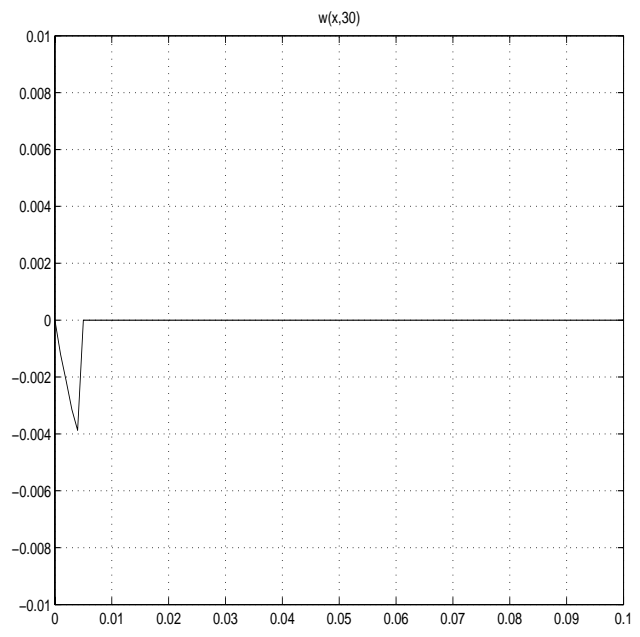

(c) $w(x, t), \quad 0 \leq x \leq 1, \quad t=30$

Figure 4.1: Snapshots of (a) $u(\cdot, t)$, (b) $v(\cdot, t)$ and (c) $w(\cdot, t)$ for $t=30$ in Example 4.1. The reader may observe quite chaotic oscillatory behavior of $u$ and $v$, but the contrary for $w$, which is a consequence of Corollary 4.1. 
the map $G_{\eta} \circ F_{\alpha, \beta}: I \rightarrow I$ is chaotic on an invariant interval, then property (4.25) should still hold for a large class of initial data $u_{0}$ and $v_{0}$. This still needs to be proved.

(c) The proof of Corollary 4.1 essentially says that there will be no chaos in the $w(\cdot, t)$ variable as $t \rightarrow \infty$. In order to have (4.27), the nonlinear boundary condition (1.3) must be replaced by

$$
\begin{aligned}
w_{x}(1, t)= & {\left[\alpha-3 \beta w^{2}(1, t)\right] w_{t}(1, t) } \\
& -\gamma w(1, t), \quad t>0,
\end{aligned}
$$

with everything else remaining unchanged. The analysis of the boundary condition (4.28) is yet to be carried out.

Acknowledgements: This work was completed while G. Chen was visiting the Center for Theoretical Sciences (CTS) of National Tsing Hua University in Hsinchu, Taiwan during July 2001. He wishes to thank CTS for the hospitality and the support for the visit.

\section{References}

Chen, G. \& Zhou, J. [1993] Vibration and Damping in Distributed Systems, Vol. I: Analysis, Estimation, Attenuation and Design (CRC Press, Boca Raton, FL), p. 15.

Chen, G., Hsu, S.B. \& Zhou, J. [1996] "Linear superposition of chaotic and orderly vibrations on two serially connected strings with a van der Pol joint", Int. J. Bifurcation and Chaos 6, 1509-1527.

Chen, G., Hsu, S.B. \& Zhou, J. [1998a] "Chaotic vibrations of the one-dimensional wave equation due to a self-excitation boundary condition, Part I, controlled hysteresis", Trans. Amer. Math. Soc. 350, 4265-4311.

[1998b] "Ibid, Part II, energy injection, period doubling and homoclinic orbits", Int. J. Bifurcation \& Chaos 8, 423-445. [1998c] "Ibid, Part III, natural hysteresis memory effects", Int. J. Bifurcation \& Chaos 8, 447470.

[1998d] "Snapback repellers as a cause of chaotic vibration of the wave equation with a van der Pol boundary condition and energy injection at the middle of the span", J. Math. Phys. 39, 64596489 .

Chen, G., Huang, T., Juang, J. \& Ma, D. [2001] "Unbounded growth of total variations of snapshots of the $1 \mathrm{D}$ linear wave equation due to the chaotic behavior of iterates of composite nonlinear boundary reflection relation", in Control of Nonlinear Distributed Parameter System, Chen, G., Lascieka, I., \& Zhou, J., ed., Lecture Notes in Pure and Applied Mathematics Series \#218 (Marcel Dekker, New York), 15-42.

Devaney, R.L. [1989] An Introduction to Chaotic Dynamical Systems (Addison-Wesley, New York).

Huang, T. \& Chen, G. [2001] "Chaotic behavior of interval maps as characterized by unbounded growth of total variations of their $n$-th iterates as $n \rightarrow \infty$ ", preprint.

Juang, J. \& Shieh, S.-F. [2001] "Interval maps, total variation and chaos", preprint. 